\title{
Intervención educativa sobre infecciones de transmisión sexual en adolescentes del tercer año de secundaria del Colegio “Los Jazmines de Naranjal” de Lima, 2014
}

\author{
Silvia E. Segura Zuloaga ${ }^{1}$, Juan P. Matzumura Kasano², Hugo Gutiérrez Crespo ${ }^{3}$
}

\section{RESUMEN:}

Objetivo: Determinar la eficacia de una intervención educativa para elevar los conocimientos sobre las Infecciones de Transmisión Sexual (ITS) en adolescentes del tercer año de educación Secundaria del colegio "Los Jazmines de Naranjal". Material y Métodos: Estudio pre experimental, prospectivo y longitudinal con una muestra de 95 adolescentes. Al inicio, se verificó con un pre-test un inadecuado nivel de conocimientos sobre Infecciones de Transmisión Sexual. Dicho instrumento estuvo dividido en cuatro dimensiones (definición, signos y síntomas, transmisión y prevención). Se implementó una intervención educativa de seis sesiones con estrategias de aprendizaje activo, con un post-test al finalizar ésta. Se utilizó el análisis descriptivo, distribución de frecuencias y porcentajes, y la Prueba de Wilconxon para determinar la eficacia de la intervención.

Resultados: De la muestra, $56 \%$ y $44 \%$, son de adolescentes sexo femenino y masculino respectivamente, con edades entre 12 a 17 años. El 11.3\% de las mujeres y el 31.8\% de los varones eran sexualmente activos. Como resultado de la intervención educativa, el porcentaje de adolescentes que obtuvieron un alto nivel de conocimientos sobre ITS, incrementó en las cuatro dimensiones (Definición: 91\%, Transmisión: 100\%, Signos y Síntomas: 98\%, Prevención: 100\%).

Conclusiones: La intervención educativa logró mejorar los conocimientos sobre las Infecciones de Transmisión Sexual en los adolescentes, con resultados estadísticamente significativos. (Horiz Med 2015; 15(4): 11-20)

Palabras clave: adolescentes, ITS, intervención educativa. (Fuente: DeCS BIREME)

Educational intervention on sexually transmitted infections in adolescents of 3rd year of Secondary School “Los Jazmines de Naranjal” in Lima, 2014

\section{ABSTRACT}

Objective: Determine the effectiveness of an educational intervention in order to increase the knowledge about Sexually Transmitted Infections (STI) in third-grade adolescents of "Los Jazmines de Naranjal" High School.

Material and Methods: A pre-experimental, prospective and longitudinal study with a sample of 95 adolescents. At the beginning based on a Pre-test, an inadequate level of knowledge about Sexually Transmitted Infections was verified, that test was divided in four dimensions (definition, signs and symptoms, transmission and prevention). An educational intervention was implemented, with six sessions, where active learning strategies were used, and with a Post-test at the end of this. Descriptive analysis, frequency distribution and percentages, and the Wilconxon Test, were used to determine the intervention effectiveness.

Results: In the sample, the $56 \%$ and $44 \%$ were female and male respectively, the age range was from 12 to 17 years, and $11.3 \%$ of the females and $31.8 \%$ of the men were sexually active. As a result of the educational intervention, the portion of adolescents with high level of STI knowledge increased in the four dimensions (definition: $91 \%$, transmission: 100\%, signs and symptoms: $98 \%$, prevention: $100 \%$ ).

Conclusions: The educational intervention improved the knowledge about sexually transmitted infections in adolescents. This improvement is statistically significant. (Horiz Med 2015; 15(4): 11-20)

Key words: adolescents, STI, educational intervention. (source: MeSH NLM)

Licenciada en Obstetricia. Responsable de Participación Comunitaria y Vida Sana del Área de Promoción de la Salud del Hospital de Huaycán.

2 Médico Ginecólogo Obstetra. Doctor en Medicina. Docente de Postgrado de la Facultad de Medicina de la USMP. Miembro del Consejo Directivo del Sistema de Certificación y Recertificación del Colegio Médico del Perú. Presidente de la Federación Latinoamericana de Sociedades de Climaterio y Menopausia (FLASCYM).

3 Obstetra. Magíster en Docencia e Investigación en Salud. Coordinador Académico de la Sociedad Peruana de Obstetricia y Ginecología (SPOG). 


\section{INTRODUCCIÓN}

Según la Organización Mundial de la Salud, se considera adolescentes a las personas con edades comprendidas entre 10 a 19 años. Según la UNICEF, en América Latina y el Caribehay más de 100 millones de adolescentes entre 10 y 18 años de edad, y se estima que la población adolescente en el Perú corresponde al $19.3 \%$ de la población total $(1,2)$.

Generalmente, los adolescentes son un grupo muy saludable; sin embargo, muchos de ellos en la edad adulta sufren de enfermedades graves pero prevenibles que comienzan en la adolescencia. Tal es el caso de las Infecciones de Transmisión Sexual (ITS), como el Virus de Inmunodeficiencia Humana (VIH), el Síndrome de Inmunodeficiencia Adquirida (SIDA), Hepatitis B, la infección por el Virus del Papiloma Humano y muchas más infecciones. Todo lo anterior es origen de afecciones graves o de muerte prematura en etapas posteriores de la vida $(1,3)$.

Aproximadamente el $50 \%$ de las mujeres entre 15 a 24 años en algunos países de Centroamérica, inició relaciones sexuales a los 15 años de edad. En nuestro país, el mayor número de adolescentes empieza aproximadamente a los 14 años, exponiéndolos a prácticas de riesgo en la esfera sexual, si es que desconocen los riesgos de una relación sexual no responsable y de las ITS $(4,5)$.

Las Infecciones de Transmisión Sexual afectan a uno de cada diez jóvenes de entre 15 y 24 años a nivel mundial. En las Américas, éstas afectan a uno de cada 20 adolescentes cada año y las más frecuentes son: Clamidia, Gonorrea, Sífilis y Tricomonas. Sin tratamiento a largo plazo, las ITS aumentan el riesgo de desarrollar cáncer de cuello uterino y de enfermedad inflamatoria pélvica, siendo responsables de la mitad de los casos de infertilidad $(1,3,6)$. En nuestro país, la prevalencia de ITS en adolescentes es del 18\%. Asimismo, el 44,3\% de las adolescentes no conocen sobre las ITS, y de las que conocen, solo el $13,3 \%$ conoce algún síntoma asociado con las ITS (5). Es decir que hay un alto porcentaje de adolescentes con desconocimiento sobre el tema.
En cuanto al VIH, según la Organización Mundial de la Salud, una séptima parte de las nuevas infecciones en todo el mundo se producen en la adolescencia y más de dos millones de adolescentes entre 10 y 19 años viven con el VIH. En el Perú, según el Ministerio de Salud, más del $50 \%$ de los casos de SIDA se registran en personas de 20 a 34 años de edad, lo que nos indica que la transmisión ocurrió en la adolescencia $(7,8)$.

Con todo lo anteriormente expuesto, se hace evidente que entre los problemas de salud que afectan a la adolescencia o en etapas posteriores de su vida se encuentran las Infecciones de Transmisión Sexual, incluido el VIH y el SIDA.

Estas surgen como consecuencia de las conductas de riesgo, ya sea por la falta o inadecuada información respecto al tema, lo que puede dejar graves secuelas para la vida sexual de los adolescentes, afectando su calidad de vida.

Mundialmente, las ITS representan un serio problema en la salud y tienen un fuerte impacto económico y social; por ello, es fundamental utilizar todas las herramientas disponibles para realizar una prevención adecuada y la promoción de prácticas saludables. Su control es decisivo para mejorar la salud reproductiva de toda la población y representa uno de los grandes desafíos de la salud pública contemporánea.

La educación a los adolescentes sobre las Infecciones de Transmisión Sexual es trascendental, ya que mejora los conocimientos que tienen sobre aquellos problemas de salud y permite desterrar los mitos y/ o la inadecuada información respecto a este tema. Además, orienta al adolescente a fortalecer el desarrollo de hábitos saludables, fomentando su pensamiento crítico para la toma de decisiones en su salud.

De esa manera, los adolescentes tendrán la capacidad para tomar decisiones que permitan demorar el inicio de las relaciones sexuales, disminuir el número de parejas sexuales, evitar conductas de riesgo y tomar medidas de prevención contra el VIH y otras ITS. 
Al respecto, un estudio preexperimental en 221 adolescentes que recibieron una intervención educativa realizado en Cuba, mostró que el $97.7 \%$ logró elevar su conocimiento sobre las infecciones consideradas de transmisión sexual, quedando vacía la categoría de nivel bajo. El 100\% respondió correctamente sobre la forma de infectarse con una ITS (VIH/SIDA); igualmente, se elevó el conocimiento sobre los síntomas inmediatos a la infección, las medidas de prevención y el uso de preservativo. Se concluyó que la intervención propuesta fue efectiva (9).

Por otro lado, en un estudio cuasi-experimental también realizado en Cuba en 200 adolescentes para identificar los conocimientos, las fuentes de información y los comportamientos de los adolescentes y así implementar acciones educativas que incrementen sus conocimientos y promover la adopción de estilos de vida más saludables, se encontró que después de la intervención, se elevaron de forma significativa los conocimientos generales (de $73 \%$ a $92 \%$ ) y clínicos (de $15 \%$ a $72 \%$ ), así como los relacionados a la transmisión (de 92\% a 94\%) y la prevención (de 77 a $80 \%$ ). Concluyendo que la implementación de una estrategia de capacitación sobre el VIH, logró mejorar el conocimiento de este aspecto en ese grupo de adolescentes (10).

Es por ello que las intervenciones que abordan estas necesidades de aprendizaje pueden mejorar la calidad de vida de los adolescentes e impulsar una nueva generación de adultos responsables y productivos que puedan contribuir al progreso de sus comunidades y que a largo plazo podrían cambiar el perfil epidemiológico del Perú.

El presente estudio adquiere relevancia por la aplicación de una intervención educativa, donde se proporcionará información adecuada y actualizada con el fin de incrementar los conocimientos hacia la prevención de las Infecciones de Transmisión Sexual y la promoción de prácticas saludables.

Así mismo, el futuro de la epidemia de VIH está en manos de los jóvenes, porque los comportamientos que adopten ahora y los que mantengan durante toda su vida sexual determinarán la evolución de la epidemia en las próximas décadas, su comportamiento dependerá en gran parte de la información y los conocimientos que la generación actual de adultos brinde a los adolescentes (11-16). El objetivo general fue determinar la eficacia de una intervención educativa para elevar los conocimientos sobre de Infecciones de Transmisión Sexual (ITS) en adolescentes del tercer año de educación Secundaria.

\section{MATERIAL Y MÉTODOS}

Se realizó un estudio de enfoque cuantitativo, preexperimental, prospectivo y longitudinal. La muestra fue de 95 adolescentes que cursaban el tercer año de educación Secundaria del colegio "Los Jazmines de Naranjal” en el 2014. Se excluyó a los adolescentes que no obtuvieron el consentimiento informado de sus padres o tutores.

Se elaboró un cuestionario de 24 preguntas, cuatro de ellas correspondían a datos generales y las demás a las dimensiones de definición, signos y síntomas, transmisión y prevención de las Infecciones de Transmisión Sexual.

Se realizó una prueba piloto a 20 adolescentes, lo cual permitió mejorar el planteamiento de las preguntas.

Para la consistencia interna del cuestionario, se utilizó el método de Kuder Richardson, obteniéndose KR20 $=0.76$, que evidenció la confiabilidad del instrumento.

Se realizó una prueba de congruencia de juicios por Coeficiente de Aiken entre los cinco jueces expertos, con un grado de concordancia significativa de 0.9. Para medir el nivel de conocimientos de todo el cuestionario se aplicó una escala de calificación que constó de tres niveles: Alto, Medio y Bajo, según Estaninos (Nivel Alto $=18$ a 20, Nivel Medio $=$ 11 a 17, Nivel Bajo < 11).

Del mismo modo, se aplicó este procedimiento para cada una de las dimensiones (definición, signos y síntomas, transmisión y prevención). 
La investigación se llevó a cabo en 4 etapas:

1. Etapa de diagnóstico de conocimientos: Se aplicó el cuestionario para medir el nivel de conocimientos sobre Infecciones de Transmisión Sexual (ITS) en los adolescentes del tercer año de educación Secundaria (pretest).

2. Elaboración de la intervención educativa:

Se confeccionó una intervención de seis sesiones de trabaj 0 , cuyos temas abarcaron; el conocimiento del aparato reproductivo femenino y masculino; infecciones de transmisión sexual curables (Clamidia, Gonorrea, Tricomoniasis, Enfermedad Inflamatoria Pélvica, Sífilis, Pediculosis Púbica); infecciones de transmisión sexual no curables (Herpes, Verrugas Genitales - VPH, Hepatitis B, VIH/SIDA); actitudes y prácticas saludables para la prevención de las ITS y uso correcto del preservativo masculino.

3. Etapa de aplicación de la intervención:

Se aplicó una intervención educativa a los adolescentes del tercer año de educación Secundaria sobre aspectos determinados de las Infecciones de Transmisión Sexual durante cinco meses, teniendo como punto de partida el diagnóstico inicial (pre-test) para dar respuesta a las necesidades de aprendizaje identificadas y así elevar sus conocimientos en los diferentes aspectos sobre las ITS, donde las respuestas no fueron satisfactorias.

Se aplicó la intervención a cuatro secciones de unos 25 adolescentes con el objetivo de facilitar la aplicación de las técnicas participativas y lograr una asimilación óptima de los contenidos impartidos.

Se utilizaron técnicas pedagógicas activas como: técnicas de investigación (Iluvia de ideas), expositivas (exposición teórica con discusión de análisis de casos) (Imagen 1), para el desarrollo de habilidades (sociodramas, talleres vivenciales) (Imagen 2), talleres prácticos (Imagen 3) y un asesoramiento y difusión de vídeos y experiencias a través de una red social a lo largo de la intervención educativa, con la participación de los adolescentes en todo el proceso.

Las actividades de la intervención fueron impartidas por la autora de la investigación.

4. Etapa de evaluación de la intervención:

Se aplicó nuevamente el cuestionario a los mismos estudiantes para medir la modificación favorable de los conocimientos sobre ITS, una vez culminadas las sesiones de la intervención educativa (post-test).

Los datos del pre y post-test fueron analizados para realizar un análisis comparativo y finalmente se discutió los resultados llegando a las conclusiones de la investigación y las recomendaciones respectivas.

Se utilizó SPSS 22.0,EPIDAT3.1 y Microsoft Office Excel 2010 para el análisis descriptivo, para el análisis de datos intervinientes se usó la distribución de frecuencias y porcentajes, y posteriormente se aplicó la Prueba de Wilconxon, para muestras relacionadas, que es una prueba no paramétrica, ya que la muestra no es de distribución normal.

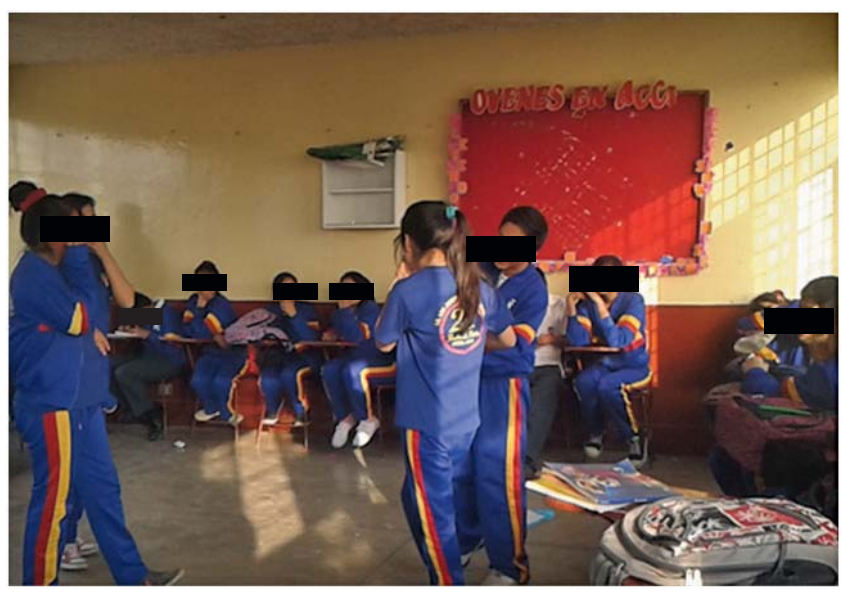

Imagen 1. Adolescentes desarrollando un sociodrama 


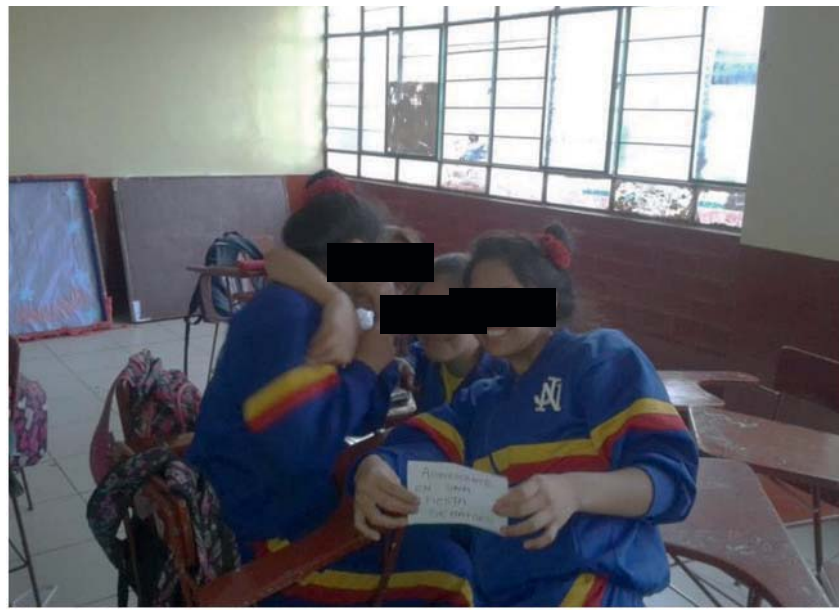

Imagen 2. Adolescentes desarrollando una discusión de casos

\section{RESULTADOS}

Los resultados de los datos generales de los adolescentes se muestran en la Tabla 1. En la Tabla 2 , se especifica la proporción de adolescentes que son sexualmente activos por sexo.

En la Tabla 3, se compara el nivel de conocimientos sobre ITS antes (pre-test) y después (post-test) de la intervención educativa por cada una de las dimensiones, mientras que en la Figura 1 se muestra la comparación del nivel de conocimientos sobre ITS antes y después de la intervención educativa del cuestionario en su totalidad.

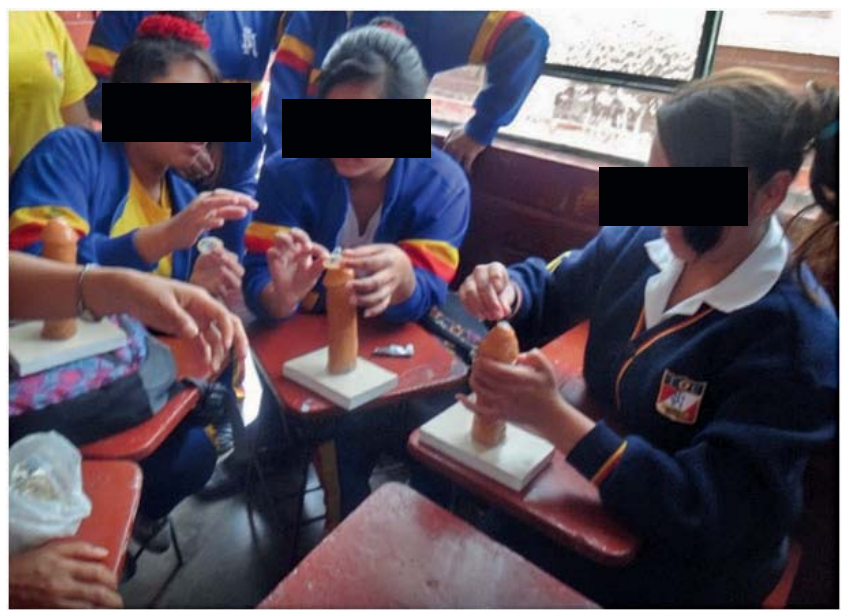

Imagen 3. Adolescentes en un taller sobre el uso correcto del preservativo masculino

En la Tabla 4 se muestra la Prueba de Rangos de Wilcoxon para pruebas no paramétricas, donde se muestra que hubo un incremento estadísticamente significativo del nivel de conocimientos sobre ITS.

En cuanto a los datos generales de los adolescentes encuestados, la primera variable interviniente evaluada fue la edad, evidenciándose que la mayoría (71\%) tiene 14 años. La segunda variable interviniente es el sexo, siendo el $56 \%$ (53) de sexo femenino y el $44 \%$ (42) de sexo masculino (Tabla 1$)$.

Tabla 1. Datos generales de los adolescentes del tercer año de educación Secundaria del Colegio “Los Jazmines de Naranjal”. 2014.

\begin{tabular}{|c|c|c|c|}
\hline VARIABLE & CRITERIO DE MEDICIÓN & $\mathbf{N}^{0}$ & $\%$ \\
\hline \multirow[t]{6}{*}{ Edad } & 12 años & 1 & $1 \%$ \\
\hline & 13 años & 7 & $7 \%$ \\
\hline & 14 años & 67 & $71 \%$ \\
\hline & 15 años & 15 & $16 \%$ \\
\hline & 16 años & 4 & $4 \%$ \\
\hline & 17 años & 1 & $1 \%$ \\
\hline \multirow[t]{2}{*}{ Sexo } & Femenino & 53 & $56 \%$ \\
\hline & Masculino & 42 & $44 \%$ \\
\hline
\end{tabular}

Fuente: Elaboración propia basada en un cuestionario, el número total de adolescentes fue de 95. 
En relación al inicio de las relaciones sexuales, el 23\% (22) afirmó haber iniciado sus relaciones sexuales al momento de la investigación, de los cuales la mayoría fueron varones (Tabla 2).

Tabla 2. Datos generales de los adolescentes del tercer año de educación Secundaria del Colegio "Los Jazmines de Naranjal". 2014.

\begin{tabular}{|crrrr|}
\hline Inicio de las & \multicolumn{2}{c}{ Sexo } & Total & $\%$ \\
relaciones sexuales & F & M & & \\
SI & 6 & 16 & 22 & $23 \%$ \\
NO & 47 & 26 & 73 & $77 \%$ \\
TOTAL & 53 & 42 & 95 & $100 \%$ \\
\hline
\end{tabular}

Fuente: Elaboración propia basada en un cuestionario, el número total de adolescentes fue de 95.

Se halló que del total de varones que inició sus relaciones sexuales, el 50\% fue a los 14 años de edad, seguido del 31\% a los 13 años y del 19\% a los 12 años. Del total de mujeres que inició sus relaciones sexuales, el $50 \%$ de ellas lo inició a los 13 años de edad, seguido del 33\% a los 14 años y del $17 \%$ a los 15 años.

En el pre-test, en cuanto a la dimensión definición, se encontró que la mayor parte de los adolescentes (79\%), obtuvo un nivel de conocimiento medio, sólo el $14 \%$ un nivel de conocimiento alto y el $7 \%$ un nivel de conocimiento bajo. Respecto a la transmisión, el mayor porcentaje de adolescentes (95\%) obtuvo un nivel de conocimiento alto; el resto $(5 \%)$ un nivel de conocimiento medio.
En referencia a los signos y síntomas, la mayor parte de adolescentes (60\%), obtuvo un nivel de conocimiento medio, el $35 \%$ un nivel de conocimiento alto y el $5 \%$ un nivel de conocimiento bajo. Finalmente en cuanto a la prevención, los adolescentes en su mayoría (68\%) obtuvieron un nivel de conocimiento medio, seguido de un 31\% de nivel de conocimiento bajo y sólo el $1 \%$ un nivel de conocimiento alto.

En el post-test, en relación a la dimensión definición, la mayoría de los adolescentes $(96 \%)$ obtuvo un nivel de conocimiento alto; es decir hubo un incremento del $82 \%$ en comparación del pre-test; el $4 \%$ obtuvo un nivel de conocimiento medio y ninguno obtuvo un nivel de conocimiento bajo.

Respecto a la transmisión, la totalidad de adolescentes (100\%) obtuvo un nivel de conocimiento alto. Respecto a los signos y síntomas, la mayor parte de adolescentes (98\%), obtuvo un nivel de conocimiento alto, el $2 \%$ un nivel de conocimiento medio y ninguno obtuvo un nivel de conocimiento baj o; es decir, que en comparación al pre-test, hubo un incremento del $53 \%$ en el nivel alto. Finalmente, en cuanto a prevención, todos los adolescentes $(100 \%)$ obtuvieron un nivel de conocimiento alto, es decir que en comparación al pre-test hubo un incremento del 99\% (Tabla 3).

Tabla 3. Nivel de conocimientos sobre infecciones de transmisión sexual por dimensiones en el pre y post-test de los adolescentes del tercer año de educación Secundaria del Colegio “Los Jazmines de Naranjal”. 2014.

\begin{tabular}{|c|c|c|c|c|c|}
\hline \multirow[t]{2}{*}{ Dimensiones } & \multirow{2}{*}{$\begin{array}{c}\text { Nivel de } \\
\text { conocimiento }\end{array}$} & \multicolumn{2}{|c|}{ Pre-test } & \multicolumn{2}{|c|}{ Post-test } \\
\hline & & $\mathrm{N}^{0}$ & $\%$ & $\mathrm{~N}^{0}$ & $\%$ \\
\hline \multirow[t]{3}{*}{ Definición } & Bajo & 7 & $7 \%$ & 0 & $0 \%$ \\
\hline & Medio & 75 & $79 \%$ & 4 & $4 \%$ \\
\hline & Alto & 13 & $14 \%$ & 91 & $96 \%$ \\
\hline \multirow[t]{3}{*}{ Transmisión } & Bajo & 0 & $0 \%$ & 0 & $0 \%$ \\
\hline & Medio & 5 & $5 \%$ & 0 & $0 \%$ \\
\hline & Alto & 90 & $95 \%$ & 95 & $100 \%$ \\
\hline \multirow[t]{3}{*}{ Signos y Síntomas } & Bajo & 5 & $5 \%$ & 0 & $0 \%$ \\
\hline & Medio & 57 & $60 \%$ & 2 & $2 \%$ \\
\hline & Alto & 33 & $35 \%$ & 93 & $98 \%$ \\
\hline \multirow[t]{3}{*}{ Prevención } & Bajo & 29 & $31 \%$ & 0 & $0 \%$ \\
\hline & Medio & 65 & $68 \%$ & 0 & $0 \%$ \\
\hline & Alto & 1 & $1 \%$ & 95 & $100 \%$ \\
\hline
\end{tabular}

Fuente: Elaboración propia basada en un cuestionario, el número total de adolescentes fue de 95. 
En la investigación entre el pre-test y el post-test del cuestionario con todas sus dimensiones, se encontró que hubo un incremento del $77 \%$ de adolescentes que alcanzaron un alto nivel de conocimientos sobre las Infecciones de Transmisión Sexual, sòlo un $23 \%$ alcanzó un nivel medio disminuyendo respecto al pre-test y ningún adolescente obtuvo un nivel bajo (Figura 1).

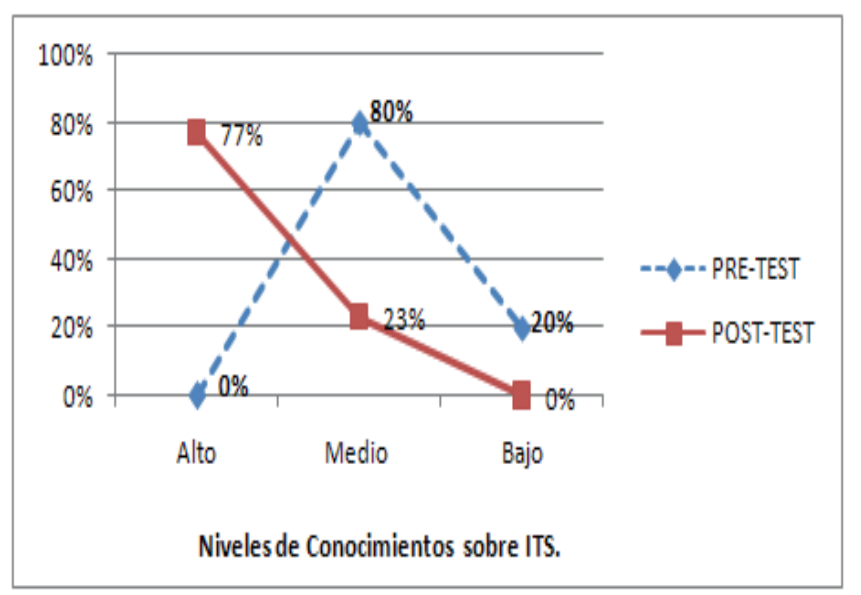

Figura 1. Comparación del nivel de conocimientos sobre infecciones de transmisión sexual en el pre-test y en el post-test de los adolescentes del tercer año de educación Secundaria del Colegio "Los Jazmines de Naranjal". 2014.
Para determinar si la intervención educativa fue eficaz, se debe obtener una diferencia significativa entre la medición del post-test y la medición del pre-test. Para ello, se utilizó la Prueba de Wilcoxon para comparar las dos mediciones y determinar si la diferencia no se produjo al azar y que sea estadísticamente significativa.

Por ello, se formula que:

Ho: Medición post-test - Mediciónpre-test $=0$

Donde Ho significa que no hay diferencia significativa en el nivel de conocimientos antes (post-test) y después (pre-test) de la intervención educativa. Si $\mathrm{p} \leq 0.05$ se rechaza la Ho.

La Prueba de Rangos de Wilcoxon muestra que los 95 alumnos que formaron parte del estudio incrementaron su nivel de conocimientos después de la intervención educativa, lo que evidencia que la intervención educativa fue eficaz $(p=0.000)$ (Tabla 3).

Es decir, se rechaza la Ho y se considera que la diferencia es estadísticamente significativa.

Tabla 4. Prueba de Wilcoxon para el nivel de conocimientos sobre infecciones de transmisión sexual entre el pre-test y post-test de los adolescentes del tercer año de educación Secundaria del colegio “Los Jazmines de Naranjal”. 2014.

\begin{tabular}{|llrrr}
\hline $\begin{array}{l}\text { Nivel de conocimientos } \\
\text { pot-test }\end{array}$ & Rangos negativos & $0^{\mathrm{a}}$ & $\begin{array}{c}\text { Rango } \\
\text { promedio }\end{array}$ & $\begin{array}{c}\text { Suma de } \\
\text { rangos }\end{array}$ \\
$\begin{array}{l}\text { Nivel de conocimientos } \\
\text { pre-test }\end{array}$ & $\begin{array}{l}\text { Rangos positivos } \\
\text { Empates }\end{array}$ & $95^{\mathrm{b}}$ & 48,00 &, 00 \\
& $0^{\mathrm{c}}$ & & 4560,00 \\
& Total & 95 & & \\
\hline
\end{tabular}

a. Nivel de Conocimiento Post-test < Nivel de Conocimiento Pre-test

b. Nivel de Conocimiento Post-test $>$ Nivel de Conocimiento Pre-test

c. Nivel de Conocimiento Post-test $=$ Nivel de Conocimiento Pre-test Estadísticos de contraste ${ }^{b}$

Nivel de conocimientos post-test Nivel de conocimientos pre-test 


\section{DISCUSIÓN}

El incremento del VIH/SIDA y otras ITS en adolescentes y jóvenes menores de 18 años se ha relacionado con escasos conocimientos acerca del tema; la no identificación de factores protectores para prevenir la infección, el inicio temprano de las relaciones sexuales, múltiples parejas, la falta de protección en contactos ocasionales y el poco uso del preservativo (12)

En el estudio se identifica que el $23 \%$ de los adolescentes encuestados ya había iniciado sus relaciones sexuales al momento de la investigación. En cuanto a los adolescentes de sexo masculino, el $50 \%$ inició sus relaciones sexuales a los 14 años y la mayor parte de las adolescentes (50\%) a los 13 años. Esto concuerda con el estudio realizado por Concepción en el año 2011, donde el 21\% de los adolescentes que participaron en la investigación habían tenido relaciones sexuales precoces (9). En esta investigación, el inicio de las relaciones sexuales fue más precoz en adolescentes del sexo femenino que en los varones. Según el ENDES 2013, se observa que el $5.7 \%$ de mujeres encuestadas afirmaron que habían tenido su primera relación sexual antes de los 15 años (5).El inicio de las relaciones sexuales a temprana edad y el número de parejas sexuales, puede traer como consecuencia un mayor riesgo de contraer una infección por contacto sexual, contribuyendo a la incidencia de alguna Infección de Transmisión Sexual.

De acuerdo con estos resultados, el inicio de la vida sexual en la población objetivo está ocurriendo precozmente, condición que refuerza el planteamiento que llevó a diseñar el estudio.

En la medición de los conocimientos en general sobre Infecciones de Transmisión Sexual en el pretest, se observó que el $80 \%$ de los adolescentes obtuvo un nivel de conocimiento medio sobre las ITS, mientras que el $20 \%$ obtuvo un conocimiento bajo y ninguno obtuvo un nivel de conocimiento alto. Es decir, que el $100 \%$ de los adolescentes no contaban con un conocimiento óptimo acerca de las ITS. Esto es coincidente con un estudio realizado por Fernández en el año 2010, la cual concluyo que el $79 \%$ de adolescentes referían no tener conocimientos sobre ITS (10).

Para la medición después de la intervención (posttest) para el nivel de conocimientos en general sobre Infecciones de Transmisión Sexual, se logró que el $77 \%$ de los adolescentes obtuvieran un nivel de conocimiento alto sobre las ITS, mientras que el $23 \%$ obtuvo un conocimiento medio y ninguno obtuvo conocimiento bajo. Es decir, que hubo un incremento del $77 \%$ de adolescentes que obtuvieron un nivel alto de conocimientos sobre ITS y ocurrió también un descenso del $53 \%$ de adolescentes que lograron un nivel medio de los conocimientos sobre ITS, puesto que porcentaje restante pasó a tener un alto nivel de conocimientos. Asimismo, hubo un descenso del total de adolescentes con nivel bajo de conocimientos sobre ITS, no quedando ninguno dentro de ese nivel. Por ello se observa que casi todos lograron mejorar sus conocimientos sobre ITS. Estos resultados concuerdan con el estudio realizado por Pérez en el año 2012, donde uno de los resultados posteriores a la intervención fue que el $\mathbf{9 7 . 7 \%}$ de los adolescentes logró elevar su conocimiento sobre las ITS (11).

Para la medición inicial antes de la intervención (pre-test) para el nivel de conocimientos por dimensiones, se encontró que para la dimensión Definición de las ITS, el 79\% obtuvo un conocimiento medio, lo que refleja que no existe suficiente información respecto a las definiciones de las Infecciones de Transmisión Sexual, y este indicador es importante porque si los adolescentes conocen adecuadamente del tema, podrán diferenciarlas de otras patologías, identificar los factores de riesgo y tomar las medidas de prevención adecuadas para evitar estas enfermedades.

En cuanto al indicador transmisión, se encontró que la mayoría de adolescentes (95\%) obtuvo un nivel de conocimiento alto, y la importancia de esta dimensión radica en que si los adolescentes conocen las vías de transmisión de las Infecciones de Transmisión Sexual, al evitarlas se podría disminuir la incidencia de cualquiera de estas ITS 
en nuestro país. Además, que la sensibilización respecto a este tema producirá en ellos una mayor responsabilidad para utilizar medidas preventivas en bien de su salud. Por ello, se debe lograr que el $100 \%$ de ellos conozca las vías de transmisión de las ITS. Respecto a la dimensión de signos y síntomas, se encontró que el $60 \%$ de adolescentes tuvo un nivel de conocimientos medio, el $35 \%$ un nivel alto y el $1 \%$ un nivel bajo, lo cual nos alerta porque más de la mitad de adolescentes no puede reconocer los signos y síntomas de las ITS de manera adecuada y por ende no buscará atención oportuna si es que presentaran una ITS. Finalmente en la medición inicial de la dimensión prevención, se encontró que el $68 \%$ obtuvo un nivel medio, el $31 \%$ un nivel bajo y el $1 \%$ un nivel alto de conocimientos, lo que nos demuestra que el $99 \%$ de adolescentes no conoce adecuadamente sobre medidas de prevención para evitar la transmisión de alguna ITS; esto es alarmante, porque el conocimiento en la prevención de estas enfermedades contribuye a que el adolescente realice medidas preventivas o comportamientos saludables como son: el uso del preservativo, fidelidad o abstinencia sexual, entre otras. Todos estos datos son similares con la investigación realizada por Fernández en el año 2010, donde el mayor número de adolescentes (79), refirieron que desconocían los elementos básicos relacionados con las ITS antes de aplicar la intervención y solamente 21 respondieron que conocían sobre el tema.

En la medición final (post-test) de la dimensión definición, se observó que el $91 \%$ de adolescentes consiguió un nivel alto, aumentando en un $12 \%$ respecto al pre-test. En cuanto a la dimensión transmisión, la totalidad de adolescentes (100\%) obtuvo un nivel de conocimientos alto.

Respecto a signos y síntomas, el $98 \%$ alcanzó un nivel de conocimientos alto, incrementándose en un $63 \%$ a comparación del pre-test. Finalmente en cuanto a la dimensión de prevención, se logró que la totalidad de adolescentes (100\%) consiguieran un nivel de conocimiento alto. Esto concuerda con una investigación realizada por Cañas en el 2012, donde se observa que después de una intervención educativa, se elevaron de forma significativa los conocimientos generales (de 73\% a 92\%), clínicos (de $15 \%$ a $72 \%$ ), de transmisión (de $92 \%$ a $94 \%$ ) y de prevención (de $77 \%$ a $80 \%$ ) (12).

El obj etivo principal de este estudio fue determinar la eficacia de una Intervención Educativa para elevar los conocimientos sobre de Infecciones de Transmisión Sexual (ITS) en adolescentes del tercer año de educación secundaria y se reconoció que la intervención educativa fue eficaz, ya que se incrementó el nivel de conocimientos sobre ITS en los adolescentes y se demuestra estadísticamente que existe una diferencia significativa que logró el cambio entre las mediciones efectuadas antes (pre-test) y después (post-test) de la intervención educativa.

En conclusión, en la mayoría de adolescentes se halló un nivel medio de conocimientos respecto a las definiciones, signos y síntomas y medidas de prevención acerca de las Infecciones de Transmisión Sexual al comienzo del estudio.

Se demostró que la aplicación de este tipo de intervenciones educativas en los adolescentes contribuye a elevar los conocimientos sobre las Infecciones de Transmisión Sexual, dándose énfasis a la promoción de prácticas saludables. De esa manera, contribuye con la promoción de la salud y con su componente educativo, que es una de las funciones esenciales de la Salud Pública.

En general, puede afirmarse que, además de mejorar los conocimientos sobre las Infecciones de Transmisión Sexual en el grupo sometido a la intervención educativa, estos proyectos logran validar un modelo de intervención primaria adecuado para los adolescentes, el cual puede ser extrapolado a mayores escalas, lo que permitiría posteriormente, la perfección de los instrumentos y métodos utilizados, así como también ampliar el estudio para investigar las conductas a largo plazo y el trabajo con pares. 


\section{REFERENCIAS BIBLIOGRÁFICAS}

1. Organización Mundial de la Salud. Salud de los adolescentes [Internet]; Organización Mundial de la Salud [citado en enero de 2014]. Disponible en: http://www.who.int/topics/adolescent_ health/ es/

2. United Nations Children's Fund (UNICEF) [Internet]. Lima, Perú: Instituto Nacional de Estadística e Informática. Estado de la Niñez en el Perú. Resume Ejecutivo. 2008 [citado en enero de 2014]. Disponible en: http://www.unicef.org/peru/spanish/Resumen_ ENP_Abril_2008.pdf

3. Organización Mundial de la Salud [Internet]. 10 Datos sobre la Salud de los adolescentes 2014 [citado en febrero de 2014] Disponible en: http://www.who.int/features/factfiles/ adolescent_health/ facts/ es/ index.html

4. Organización de los Estados Americanos [Internet]. Salud Sexual y Reproductiva [citado en febrero de 2014]. Disponible en: http://www.oas.org/es/youth/Salud_Sexual_y_ Reproductiva.asp

5. Instituto Nacional de Estadística e Informática [Internet]. Encuesta Demográfica y de Salud Familiar [citado en febrero de 2014]. Disponible en:

http://www.inei.gob.pe/media/MenuRecursivo/publicaciones_ digitales/Est/Lib1151/pdf/Libro.pdf

6. Organización Mundial de la Salud [Internet]. Campañas Mundiales de Salud Pública de la OMS[citado en febrero de 2014]. Disponible en:

http://www.who.int/campaigns/aids-day/2013/es/index.html

7. Organización Mundial de la Salud [Internet]. Diez Datos sobre las Enfermedades de Transmisión sexual [citado en enero de 2014]. Disponible en:

http:// www. who.int/ features/ factfiles/ sexually_transmitted_ diseases/facts/es/index3.html

8. Ministerio de Salud del Perú [Internet]. El VIH en los adolescentes y Jóvenes [citado en enero de 2014]. Disponible en: http://www. minsa. gob. pe/ portada/ Especiales/2011/ vih/ default. asp

9. Concepción, U. Intervención Educativa sobre Infecciones de Transmisión Sexual en adolescentes preuniversitarios en la Sierpe. Revista Contribuciones a las Ciencias Sociales [Internet]. 2011. [citado en abril de 2014]. Disponible en: http://www.eumed.net/rev/cccss/16/urg.html

10. Fernández B. Intervención educativa sobre infecciones de transmisión sexual en adolescentes. Revista Médica de Pinar del Río [Internet]. 2010, Cuba [citado en enero de 2014]; 14(3): pp. 5-9. Disponible en: http://scielo.sld.cu/pdf/rpr/v14n3/rpr03310. pdf

11. Pérez R, Echevarría Y. Intervención Educativa sobre las ITS/VIH/ SIDA en adolescentes del consultorio 24 del Consejo Popular Oeste. Convención Internacional de Salud Pública [Internet]. 2012, Cuba [citado en enero de 2014]; pp. 6-8. Disponible en: http://www. convencionsalud2012. sld.cu/ index. php/ convencionsalud/2012/ paper/view/667

12. Cañas L, Menéndez R. Intervención educativa sobre el VIH con adolescentes de la Escuela Militar "Camilo Cienfuegos" de Arroyo Arenas. Revista Cubana Medicina Tropical [Internet]. 2012, Cuba. [citado en enero de 2014]; 64(1): pp. 3-4. Disponible en: http://bvs.sld.cu/revistas/mtr/vol64_1_12/mtr06112.htm

13. Achiong, A. Intervención educativa en VIH/SIDA a adolescentes del
Instituto Preuniversitario Vocacional de Ciencias Exactas "Carlos Marx", de Matanzas. Revista Cubana de Higiene y Epidemiologia [Internet]. 2011, Cuba [citado en enero de 2014]; 49(3): pp. 336-345. Disponible en: http://www.redalyc.org/articulo. oa?id=223221363003

14. Rodríguez C. Estrategia educativa sobre promoción en salud sexual y reproductiva en adolescentes y jóvenes universitarios. Revista Cubana de Salud Pública [Internet]. 2013, Cuba [citado en enero de 2014]; 39(1): pp. 161-164. Disponible en:

http: / / www.redalyc. org/articulo.oa?id=21425613009

15. Castro A. Intervención Educativa sobre Infecciones de Transmisión Sexual en adolescentes de la secundaria Fructuoso Rodríguez. Revista Habanera de Ciencias Médicas [Internet]. 2012, Cuba [citado en febrero de 2014]; 11(2): pp. 300-307. Disponible en: http:// scielo. sld.cu/ pdf/ rhcm/ v11n2/ rhcm15212. pdf

16. Badía S. Intervención Educativa sobre el Síndrome de Inmunodeficiencia Adquirida realizada en adolescentes del Instituto Técnico de Joyaba-Guatemala. Revistas Ciencias Holguín [Internet]. 2013, Cuba [citado en febrero de 2014]; 19(1). Disponible en:http:// www. ciencias. holguin.cu/ index.php/ cienciasholguin/article/view/747/689.

\section{Fuentes de financiamiento}

Este artículo ha sido financiado por los autores.

\section{Conflicto de interés}

Los autores declaran no tener ningún conflicto de interés.

\section{Correspondencia:}

Silvia E. Segura Zuloaga

Dirección: Av. J.C. Mariátegui S/N Zona "B" Huaycán - Ate

Teléfono: 964242914

Correo electrónico: silviasegura86@gmail.com 